\title{
The Identity String Field and the Sliver Frame Level Expansion
}

\author{
Theodore Erler ${ }^{1}$ \\ Institute of Physics of the ASCR, v.v.i. \\ Na Slovance 2, 182 21 Prague 8, Czech Republic
}

\begin{abstract}
We propose a modified version of the sliver-frame level expansion which gives a tool for analyzing singularities related to the identity string field. We apply this formalism to the newly discovered solutions of Masuda, Noumi, and Takahashi.
\end{abstract}

\footnotetext{
${ }^{1}$ Email: tchovi@gmail.com
} 


\section{Contents}

1 Introduction $\quad 1$

$2 \mathcal{L}^{-}$Level Expansion $\quad 2$

3 Dual $\mathcal{L}^{-}$Level Expansion $\quad 4$

4 Applications to Solutions in the $K B c$ subalgebra $\quad 13$

5 Discussion $\quad 19$

$\begin{array}{ll}\text { A Proof of Theorem 4.1 } & 20\end{array}$

\section{Introduction}

In an interesting recent paper [1], Masuda, Noumi and Takahashi (MNT) discovered an analytic solution in open string field theory which seems to describe a D-brane with negative tension (what might be called a "ghost D-brane" [4]). The solution, while interesting, is physically problematic. One possible way out, noted in [1], is that the ghost-brane solution has a term proportional to the identity string field, which might be considered singular. While there has been much discussion of singularities in analytic solutions related to the sliver state (see, for example, $[2,3,5,6,7,8]$ ), so far there has been limited discussion of singularities related to the identity string field. ${ }^{3}$ Indeed, many useful analytic solutions in open string field theory (e.g. [9, 13]) are quite "identity-like," and not obviously less singular than the ghost brane of MNT.

In this paper we hope to clarify this situation. Our results can be summarized as follows:

- For fields in the subalgebra of wedge states with insertions, we can define a version of the sliver frame level expansion called the dual $\mathcal{L}^{-}$level expansion. It is similar to the $\mathcal{L}_{0}$ level expansion of Schnabl [14], except fields are expanded around $K=\infty$ rather than $K=0$. This allows us to formulate the following criterion: A solution is too identity-like if its highest level in the dual $\mathcal{L}^{-}$level expansion is zero or positive.

- We show that solutions in the $K B c$ subalgebra $[15,16]$ can be partitioned into exactly six distinct gauge orbits, and all but two of these (the tachyon vacuum and the perturbative vacuum) are necessarily represented by solutions with unacceptable behavior with respect to the identity string field. This means, in particular,

\footnotetext{
${ }^{2}$ The solutions proposed in [1] are different from the "ghost D-brane" solutions discussed in [2,3].

${ }^{3}$ Some recent discussion of the identity string field in connection with the tachyon vacuum solution $c(1-K)$ appears in $[10,11,12]$.
} 
that there is no regular solution for the MNT ghost-brane background in the $K B c$ subalgebra.

\section{$2 \quad \mathcal{L}^{-}$Level Expansion}

In this paper we work with the subalgebra of wedge states with insertions $[14,17,18]$. This subalgebra is generated by taking sums and products of the string fields $[15,16]$

$$
K, \quad B, \quad \phi_{i}(i=1,2, \ldots) .
$$

We define $K$ and $B$ following the conventions of [9]. The string fields $\phi_{i}$ represent insertions of boundary operators $\phi_{i}(z)$ in correlation functions on the cylinder ${ }^{4}$. Explicitly they can be defined

$$
\phi_{i} \equiv f_{\mathcal{S}}^{-1} \circ \phi_{i}\left(\frac{1}{2}\right)|I\rangle
$$

where $f_{\mathcal{S}}^{-1}(z)=\tan (\pi z / 2)$ is the inverse of the sliver coordinate map [19] and $|I\rangle$ is the identity string field (which we will often write simply as " 1 " when no confusion can arise). We assume that the $\phi_{i}(z)$ s have have definite scaling dimension $h_{i}$. Recall that the string fields $K$ and $B$ satisfy the identities [15]

$$
K=Q B, \quad[K, B]=0, \quad B^{2}=0,
$$

and $K$ generates the algebra of wedge states [17], in the sense that any star algebra power of the $S L(2, \mathbb{R})$ vacuum $\Omega \equiv|0\rangle$ can be written $\Omega^{\alpha}=e^{-\alpha K}$.

Consider the reparameterization generator $\mathcal{L}^{-}$, which is the BPZ odd component of the scaling generator in the sliver coordinate frame [14, 20]:

$$
\mathcal{L}_{0} \equiv f_{\mathcal{S}}^{-1} \circ L_{0}
$$

Specifically

$$
\mathcal{L}^{-} \equiv \mathcal{L}_{0}-\mathcal{L}_{0}^{\star}
$$

where ${ }^{\star}$ denotes BPZ conjugation. The reparameterization $\mathcal{L}^{-}$generates scale transformations of the algebra of wedge states with insertions. Acting on the elementary fields $K, B$ and $\phi_{i}$, it computes (twice) the scaling dimension of the corresponding operator insertion on the cylinder:

$$
\frac{1}{2} \mathcal{L}^{-} K=K, \quad \frac{1}{2} \mathcal{L}^{-} B=B, \quad \frac{1}{2} \mathcal{L}^{-} \phi_{i}=h_{i} \phi_{i} .
$$

Then, since $\mathcal{L}^{-}$is a derivation, this defines the action of $\mathcal{L}^{-}$on the whole subalgebra.

We can decompose a string field into $\mathcal{L}^{-}$eigenstates by expanding the field as a formal power series in $K$ around $K=0$. (To do this we assume that the $\phi_{i}$ s have regular OPEs,

\footnotetext{
${ }^{4}$ See appendix A of [9] for some background on the relation between string fields and operator insertions in correlation functions on the cylinder.
} 
otherwise this expansion can produce contact divergences.) For example, consider the zero momentum tachyon ground state

$$
\sqrt{\Omega} c \sqrt{\Omega}=\frac{2}{\pi} c(0)|0\rangle,
$$

where $\sqrt{\Omega}$ is the square root of the $S L(2, \mathbb{R})$ vacuum. Expanding this around $K=0$ gives the expression

$$
\begin{aligned}
\sqrt{\Omega} c \sqrt{\Omega} & =\exp \left(-\frac{K}{2}\right) c \exp \left(-\frac{K}{2}\right) \\
& =c-\frac{1}{2}(c K+K c)+\frac{1}{8}\left(K^{2} c+2 K c K+c K^{2}\right)-\ldots .
\end{aligned}
$$

Each term in the series is an $\mathcal{L}^{-}$eigenstate. For a general state in the subalgebra of wedge states with insertions, expanding around $K=0$ produces an expression of the form

$$
\Phi=\Phi_{h_{1}}+\Phi_{h_{2}}+\Phi_{h_{3}}+\ldots \quad\left(h_{1}<h_{2}<h_{3}<\ldots\right),
$$

where $\Phi_{h_{n}}$ are eigenstates of $\mathcal{L}^{-}$:

$$
\frac{1}{2} \mathcal{L}^{-} \Phi_{h_{n}}=h_{n} \Phi_{h_{n}}
$$

This defines what we call the $\mathcal{L}^{-}$level expansion. We use "level" to refer to the $\frac{1}{2} \mathcal{L}^{-}$ eigenvalue in this expansion. Assuming that products of $\phi_{i} \mathrm{~s}$ do not produce operators of arbitrarily negative conformal dimension, the level is bounded from below, and higher level states are considered "subleading." We say "subleading" in quotes since the $\mathcal{L}^{-}$ level expansion is very formal: Each term in the expansion is proportional to the identity string field, and there is at least one sense (explained in the next section) that terms in the expansion become increasingly singular as the level is increased.

The $\mathcal{L}^{-}$level expansion can be understood as a variant of the $\mathcal{L}_{0}$ level expansion of Schnabl [14]. They are related through the formula [9]

$$
\mathcal{L}_{0}(\sqrt{\Omega} \Phi \sqrt{\Omega})=\sqrt{\Omega}\left(\frac{1}{2} \mathcal{L}^{-} \Phi\right) \sqrt{\Omega}
$$

This means that the $\mathcal{L}_{0}$ level expansion of the field $\sqrt{\Omega} \Phi \sqrt{\Omega}$ is equivalent to the $\mathcal{L}^{-}$ level expansion of the field $\Phi$; The coefficients of the eigenstates are the same in either expansion. One important difference, however, is that the eigenstates of the $\mathcal{L}_{0}$ level expansion are proportional to the $S L(2, \mathbb{R})$ vacuum rather than the identity string field. This makes it possible to calculate the energy of solutions in the $\mathcal{L}_{0}$ level expansion $[9,14,21,22]$, whereas this is impossible in the $\mathcal{L}^{-}$level expansion.

The $\mathcal{L}^{-}$level expansion is closely related to the phantom term in string field theory solutions. The phantom term between solutions $\Phi_{1}$ and $\Phi_{2}$ can be written schematically

$$
X^{\infty}\left(\Phi_{2}-\Phi_{1}\right)
$$


where $X^{\infty}$ is the boundary condition changing projector for a singular gauge transformation connecting $\Phi_{1}$ and $\Phi_{2}[3,8]$. Typically, $X^{\infty}$ is proportional to the sliver state, and when calculating contractions involving the phantom term the solutions $\Phi_{1}$ and $\Phi_{2}$ look, by comparison to $X^{\infty}$, like operator insertions on the identity string field. Moreover, in correlation functions on a very large cylinder, operator insertions with the lowest scaling dimension make the leading contribution. Therefore, inside the phantom term the solutions $\Phi_{1}$ and $\Phi_{2}$ are naturally described by the $\mathcal{L}^{-}$level expansion.

Let us make a technical comment. In order to implement the $\mathcal{L}^{-}$level expansion in the subalgebra of wedge states with insertions, we need to make some assumptions about how the string field depends on $K$. First, it must be formally an analytic function of $K$ at $K=0$, and second, the power series expansion in $K$ at $K=0$ must uniquely characterize the string field, if it exists. The first assumption is needed otherwise the expansion at $K=0$ does not necessarily produce eigenstates of $\mathcal{L}^{-}$. For example, the field $K \ln (K)$ does not have a well-defined $\mathcal{L}^{-}$level expansion. Moreover, it is known that certain singularities at $K=0$-particularly poles $[5,2,23]$ - should not be allowed for regular string fields, though it is not clear whether complete analyticity at $K=0$ is required. As for the second assumption, the $\mathcal{L}^{-}$level expansion uniquely describes the string field as long as elements of the wedge algebra are described as Laplace transforms over wedge states [24]. More exotic functions of $K$ have been constructed in [25], but it is not clear whether such states have well-defined star products. At any rate, these assumptions hold for nearly all states which are normally considered in applications, and we will assume that they hold for the remainder of the paper.

\section{$3 \quad$ Dual $\mathcal{L}^{-}$Level Expansion}

With this background, we are ready to discuss our main interest: understanding singularities in solutions related to the identity string field. Specifically, we want to answer the following question: If $\Phi$ is a string field in the subalgebra of wedge states with insertions, under what circumstances is the quantity ${ }^{5}$

$$
\operatorname{Tr}[\Phi]
$$

well-defined from the perspective of the identity string field?

To start, consider the trace of a product of local insertions

$$
\operatorname{Tr}\left[\phi_{1} \phi_{2} \ldots \phi_{n}\right] \text {. }
$$

Since the $\phi_{i}$ s are proportional to the identity string field, the trace represents a correlation function on a cylinder with vanishing area (see figure 3.1). To make sense of this, we can introduce a point-splitting regulator, separating the $\phi_{i} s$ with wedge states of non-zero width:

$$
\operatorname{Tr}\left[\phi_{1} \Omega^{\epsilon t_{1}} \phi_{2} \Omega^{\epsilon t_{2}} \ldots \phi_{n} \Omega^{\epsilon t_{n}}\right]
$$

\footnotetext{
${ }^{5}$ We denote the 1 -string vertex by the trace: $\operatorname{Tr}[\Phi] \equiv\langle I \mid \Phi\rangle$
} 


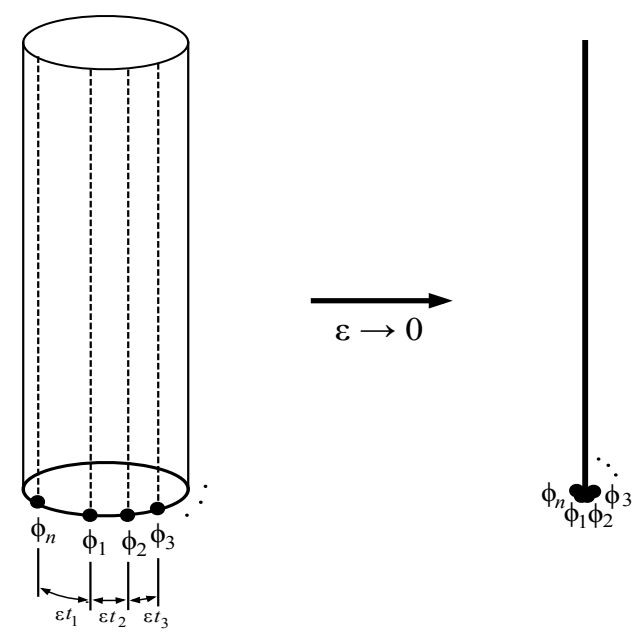

Figure 3.1: Regulating the trace of a product of local insertions.

Formally, this reproduces (3.2) in the $\epsilon \rightarrow 0$ limit. Now note that (3.3) only depends on $\epsilon$ through the total scaling dimension of the insertions:

$$
\begin{aligned}
\operatorname{Tr}\left[\phi_{1} \Omega^{\epsilon t_{1}} \phi_{2} \Omega^{\epsilon t_{2}} \ldots \phi_{n} \Omega^{\epsilon t_{n}}\right] & =\left(\frac{1}{\epsilon}\right)^{h_{1}+\ldots+h_{n}} \operatorname{Tr}\left[\epsilon^{\frac{1}{2} \mathcal{L}^{-}}\left(\phi_{1} \Omega^{t_{1}} \ldots \phi_{n} \Omega^{t_{3}}\right)\right] \\
& =\left(\frac{1}{\epsilon}\right)^{h_{1}+h_{2}+\ldots+h_{n}} \operatorname{Tr}\left[\phi_{1} \Omega^{t_{1}} \phi_{2} \Omega^{t_{2}} \ldots \phi_{n} \Omega^{t_{n}}\right],
\end{aligned}
$$

where we used reparameterization invariance of the vertex. Now let's take $\epsilon$ to zero. The limit is clearly divergent if $h_{1}+\ldots+h_{n}$ is positive. In fact, even if $h_{1}+\ldots+h_{n}=0$ the limit is singular because the answer depends on the choice of parameters $t_{1}, \ldots, t_{n}$ used to split the operators. Only if $h_{1}+\ldots+h_{n}$ is negative do we find a well defined result: $\operatorname{Tr}\left[\phi_{1} \ldots \phi_{n}\right]=0$. With an eye towards generalization, we can summarize these observations (in a somewhat technical fashion) as follows:

Theorem 3.1. If $\Phi$ is a state created by taking sums and products of local insertions $\phi_{i}$, then $\operatorname{Tr}[\Phi]$ is well defined only if the highest level in the $\mathcal{L}^{-}$level expansion of $\Phi$ is strictly negative.

At the moment this statement is somewhat uninteresting, since for a fixed set of $\phi_{i}$ s there are generally few, if any, ways to create negative level states.

We would like to find an analogue of this theorem for arbitrary fields in the subalgebra of wedge states with insertions. As a first step, consider the trace of a state with a single insertion:

$$
\operatorname{Tr}[F(K) \phi]
$$


Assuming that $F(K)$ can be written as a Laplace transform of some $f(t)$, we can write

$$
\operatorname{Tr}[F(K) \phi]=\int_{0}^{\infty} d t f(t) \operatorname{Tr}\left[\Omega^{t} \phi\right] .
$$

Note that the identity string field corresponds to the $t \rightarrow 0$ limit in the integrand. Using a reparameterization we can factor the trace out of the integration:

$$
\operatorname{Tr}[F(K) \phi]=\operatorname{Tr}[\Omega \phi] \int_{0}^{\infty} d t\left(\frac{1}{t}\right)^{h} f(t) .
$$

The integration is finite near $t=0$ if

$$
\lim _{t \rightarrow 0}\left(\frac{1}{t}\right)^{h-1} f(t)=0 .
$$

Under this condition $\operatorname{Tr}[F(K) \phi]$ is well-defined from the perspective of the identity string field. This does not mean that $\operatorname{Tr}[F(K) \phi]$ is necessarily finite. For this to be true, the full integration from $t=0$ to $\infty$ must be finite in (3.7), not just the integration in the neighborhood of $t=0$.

Let's restate condition (3.8) in a form analogous to theorem 3.1. To start, let's suppose that $f(t)$ can be written as a power series expansion around $t=0$ :

$$
\begin{aligned}
f(t) & =\sum_{n=1}^{\infty} a_{n} t^{\mu_{n}}+r(t) \\
& =a_{1} t^{\mu_{1}}+a_{2} t^{\mu_{2}}+\ldots+r(t) \quad\left(-1<\mu_{1}<\mu_{2}<\ldots .\right),
\end{aligned}
$$

where $a_{n}$ are coefficients, $\mu_{n}$ are an increasing sequence of powers (not necessarily integers), and $r(t)$ is a remainder which vanishes faster than any power at $t=0$. Condition (3.8) implies that $\mu_{1}$, the leading power in this expansion, satisfies the inequality

$$
\mu_{1}+1>h .
$$

Now suppose that we take the expansion (3.9) to define an expansion of the string field $F(K)$ :

$$
F(K)=\sum_{n=1}^{\infty} a_{n}\left(\int_{0}^{\infty} d t t^{\mu_{n}} \Omega^{t}\right)+R(K),
$$

where $R(K)$ is the Laplace transform of $r(t)$. There are two immediate problems with this expression. First, the series for $f(t)$ might only have finite radius of convergence, so (3.11) might only be an asymptotic expansion. Second, and perhaps more disturbingly, each term in (3.11) is actually a divergent string field. The integrand is completely unsuppressed for large $t$, so the formal expansion (3.11) produces a sequence of increasingly severe divergences proportional to the sliver state. However, for our purposes this is not a 
problem. Our primary interest is the identity string field, not the sliver state. If the string field is well-defined, the sliver divergences will cancel upon formal resummation of (3.11). Note that the divergent integrals in (3.11) actually define inverse powers of $K$ in the Schwinger parameterization:

$$
\int_{0}^{\infty} \frac{t^{\mu}}{\Gamma(\mu+1)} \Omega^{t} \equiv \frac{1}{K^{\mu+1}} .
$$

Therefore (3.11) can be reexpressed in the simple form

$$
F(K)=\sum_{n=1}^{\infty} \frac{b_{n}}{K^{\nu_{n}}}+R(K)
$$

where

$$
\nu_{n} \equiv \mu_{n}+1, \quad b_{n} \equiv \Gamma\left(\mu_{n}+1\right) a_{n} .
$$

It is natural to interpret (3.13) as an expansion of $F(K)$ around $K=\infty$. In fact, (3.13) looks like an expansion in $\mathcal{L}^{-}$eigenstates, in the sense that we should define

$$
\frac{1}{2} \mathcal{L}^{-} \frac{1}{K^{\nu}}=-\nu \frac{1}{K^{\nu}}
$$

by analogy to the formula $\frac{1}{2} \mathcal{L}^{-} K^{n}=n K^{n}$ for positive integer $n$. By convention, we will say that $R(K)$ has $\mathcal{L}^{-}$eigenvalue $-\infty$, since it vanishes faster than any inverse power of $K$ towards infinity. Therefore, we can interpret (3.13) as an unusual form of the $\mathcal{L}^{-}$level expansion.

For a general field $\Phi$ in the algebra of wedge states with insertions, a formal expansion around $K=\infty$ may produce an expression of the form

$$
\Phi=\Phi_{h_{1}}+\Phi_{h_{2}}+\Phi_{h_{3}}+\ldots \quad\left(h_{1}>h_{2}>h_{3}>\ldots\right),
$$

where $\Phi_{h_{n}}$ are eigenstates of $\mathcal{L}^{-}$:

$$
\frac{1}{2} \mathcal{L}^{-} \Phi_{h_{n}}=h_{n} \Phi_{h_{n}}
$$

This is what we call the dual $\mathcal{L}^{-}$level expansion. We use "level" to refer to the $\frac{1}{2} \mathcal{L}^{-}$ eigenvalue in this expansion. The level can be taken as a precise measure of how identitylike a string field is. The identity string field itself is level 0; progressively negative levels become less singular from the perspective of the identity string field, and progressively positive levels are increasingly more singular than the identity string field. Though the dual $\mathcal{L}^{-}$level expansion looks similar to the $\mathcal{L}^{-}$level expansion defined earlier, there are a number of important differences:

1) The leading level in the dual $\mathcal{L}^{-}$level expansion is the highest level. Subleading levels are increasingly negative. In the $\mathcal{L}^{-}$level expansion, the situation is opposite: The leading level is the lowest level, and subleading levels are increasingly positive. 
2) Eigenstates in the dual $\mathcal{L}^{-}$level expansion become increasingly singular from the perspective of the sliver state as the level becomes progressively negative, whereas the eigenstates in the $\mathcal{L}^{-}$level expansion become increasingly singular from the perspective of the identity string field as the level becomes progressively positive.

3) Since the dual $\mathcal{L}^{-}$level expansion respects the short distance structure of the string field, it does not produce collisions of $\phi_{i} \mathrm{~s}$ if they are not present in the state to begin with. Therefore it is not necessary to assume that the $\phi_{i}$ s have regular OPEs when implementing the dual $\mathcal{L}^{-}$level expansion, whereas this assumption appears necessary for the $\mathcal{L}^{-}\left(\right.$or $\left.\mathcal{L}_{0}\right)$ level expansion.

In a sense, the two expansions give complementary information about the string field. For example, the states

$$
\Omega \quad \Omega^{-1}
$$

look very similar in the $\mathcal{L}^{-}$level expansion as a power series around $K=0$. But in the dual $\mathcal{L}^{-}$level expansion the inverse wedge state is clearly singular since it diverges at $K=\infty$ faster than any power of $K$. On the other hand, consider the states

$$
\frac{1-\Omega}{K} \quad \frac{2-\Omega}{K}
$$

These states look similar in the dual $\mathcal{L}^{-}$expansion, but in the $\mathcal{L}^{-}$level expansion the first state is regular, while the second has a pole at $K=0$ which produces a sliver divergence. In summary, the dual $\mathcal{L}^{-}$level expansion is sensitive to singularities related to the identity string field, but not the sliver state, while precisely the opposite is true in the $\mathcal{L}^{-}$level expansion.

Let us make a technical comment: Fields in the subalgebra of wedge states with insertions are not necessarily analytic functions of $K$ around $K=\infty$. This is why noninteger powers of $K$, and the remainder $R(K)$, can appear in (3.13) while (by assumption) no such terms appear in the expansion around $K=0$. But this means that the expansion around $K=\infty$ can produce terms (for example $\frac{1}{\ln K}$ ) which are not $\mathcal{L}^{-}$eigenstates, and are not accounted for in (3.13). In this situation the field does not have a dual $\mathcal{L}^{-}$level expansion, though much of our discussion can be extended to such examples. At any rate, in most applications the string field does have a dual $\mathcal{L}^{-}$level expansion. For illustrative purposes, we have listed the $\mathcal{L}^{-}$and dual $\mathcal{L}^{-}$level expansions of some commonly encountered states in table 1.

Now let us return to the original question, which was to find an alternative expression for the regularity condition (3.8). Via equation (3.10), it is clear that (3.8) imposes a constraint on the leading power $\nu_{1}$ in the expansion of $F(K)$ around $K=\infty$ :

$$
h-\nu_{1}<0
$$

The quantity $h-\nu_{1}$ is the highest level in the dual $\mathcal{L}^{-}$level expansion of $F(K) \phi$. Recalling theorem 3.1, this suggests the general result: 


\begin{tabular}{|c|c|c|}
\hline$\Omega$ & $\mathcal{L}^{-}$expansion & dual $\mathcal{L}^{-}$expansion \\
\hline$\frac{1}{1+K}$ & $1-K+\frac{K^{2}}{2 !}-\frac{K^{3}}{3 !}+\ldots$ & $R(K)=\Omega$ \\
\hline $1+K$ & $1-K$ & $\frac{1}{K}-\frac{1}{K^{2}}+\frac{1}{K^{3}}-\ldots$ \\
\hline$\frac{1}{\sqrt{1+K}}$ & $1-\frac{1}{2} K+\frac{3}{8} K^{2}-\frac{5}{16} K^{3}+\ldots$ & $\frac{1}{K^{1 / 2}}-\frac{1}{2} \frac{1}{K^{3 / 2}}+\frac{3}{8} \frac{1}{K^{5 / 2}}-\frac{5}{16} \frac{1}{K^{7 / 2}}+\ldots$ \\
\hline$\frac{1-\Omega}{K}$ & $1-\frac{K}{2 !}+\frac{K^{2}}{3 !}-\frac{K^{3}}{4 !}+\ldots$ & $\frac{1}{K}+R(K) \quad R(K)=-\frac{1}{K} \Omega$ \\
\hline$\frac{K}{1-\Omega}$ & $1-\frac{K}{2}+\frac{K^{2}}{12}-\frac{K^{4}}{720}+\ldots$ & $K+R(K) \quad R(K)=\frac{K \Omega}{1-\Omega}$ \\
\hline
\end{tabular}

Table 1: $\mathcal{L}^{-}$and dual $\mathcal{L}^{-}$level expansions of various states in the wedge algebra.

Theorem 3.2. Let $\Phi$ be a state in the subalgebra of wedge states with insertions that admits a dual $\mathcal{L}^{-}$level expansion. Then $\operatorname{Tr}[\Phi]$ is well defined only if the highest level in the dual $\mathcal{L}^{-}$level expansion of $\Phi$ is strictly negative.

Proof. A general field in the subalgebra of wedge states with insertions can be expressed as a linear combination of states of the form

$$
\Phi=\int_{0}^{\infty} d t_{1} d t_{2} \ldots d t_{n} f\left(t_{1}, t_{2}, \ldots, t_{n}\right) \Omega^{t_{1}} \phi_{1} \Omega^{t_{2}} \phi_{2} \ldots \Omega^{t_{n}} \phi_{n} .
$$

For notational convenience we place the insertion $\phi_{n}$ at the right edge of $\Phi$. If $\Phi$ has no nontrivial insertion there, we can set $\phi_{n}=1$. Taking the Laplace transform of $f\left(t_{1}, \ldots, t_{n}\right)$ defines the function

$$
F\left(K_{1}, \ldots, K_{n}\right)=\int_{0}^{\infty} d t_{1} \ldots d t_{n} f\left(t_{1}, \ldots, t_{n}\right) e^{-t_{1} K_{1}} \ldots e^{-t_{n} K_{n}} .
$$

We can think of $F\left(K_{1}, \ldots, K_{n}\right)$ either as a function of $n$ numbers $K_{i}$, or as a function of a single string field $K$ with the understanding the index on $K_{i}$ tells us how to order $K$ 
relative to the insertions $\phi_{i}$. Taking the trace, we can reorganize the integrals over $t \mathrm{~s}$ into an integral over the total width of the cylinder $L=t_{1}+\ldots+t_{n}$ and an integral over angular parameters $\theta_{i}=t_{i} / L$ separating the insertions:

$$
\begin{aligned}
\operatorname{Tr}[\Phi] & =\int_{0}^{\infty} d t_{1} \ldots d t_{n} f\left(t_{1}, \ldots, t_{n}\right) \operatorname{Tr}\left[\Omega^{t_{1}} \phi_{1} \ldots \Omega^{t_{n}} \phi_{n}\right] \\
& =\int_{0}^{\infty} d L L^{n-1} \int d \theta_{1} \ldots d \theta_{n} \delta\left(\theta_{1}+\ldots+\theta_{n}-1\right) f\left(L \theta_{1}, \ldots, L \theta_{n}\right) \operatorname{Tr}\left[\Omega^{L \theta_{1}} \phi_{1} \ldots \Omega^{L \theta_{n}} \phi_{n}\right] .
\end{aligned}
$$

The identity string field appears in the $L \rightarrow 0$ limit of the integration. With a reparameterization we can pull the trace out of the integration over $L$ :

$\operatorname{Tr}[\Phi]=\int d \theta_{1} \ldots d \theta_{n} \delta\left(\theta_{1}+\ldots+\theta_{n}-1\right) \operatorname{Tr}\left[\Omega^{\theta_{1}} \phi_{1} \ldots \Omega^{\theta_{n}} \phi_{n}\right] \int_{0}^{\infty} d L\left(\frac{1}{L}\right)^{h_{1}+\ldots+h_{n}+1-n} f\left(L \theta_{1}, \ldots, L \theta_{n}\right)$.

We assume that this quantity is well defined only if the integration over $L$ is convergent towards $L=0$. This requires

$$
\lim _{L \rightarrow 0}\left(\frac{1}{L}\right)^{h_{1}+\ldots+h_{n}-n} f\left(L \theta_{1}, \ldots, L \theta_{n}\right)=0 .
$$

This can be equivalently stated

$$
\lim _{\alpha \rightarrow \infty} \alpha^{h_{1}+\ldots+h_{n}-n} f\left(\frac{t_{1}}{\alpha}, \ldots, \frac{t_{n}}{\alpha}\right)=0 .
$$

Now consider the limit:

$$
\begin{aligned}
\lim _{\alpha \rightarrow \infty} \alpha^{h_{1}+\ldots+h_{n}} F\left(\alpha K_{1}, \ldots \alpha K_{n}\right) & =\lim _{\alpha \rightarrow \infty} \alpha^{h_{1}+\ldots+h_{n}} \int_{0}^{\infty} d t_{1} \ldots d t_{n} f\left(t_{1}, \ldots, t_{n}\right) e^{-\alpha t_{1} K_{1}} \ldots e^{-\alpha t_{n} K_{n}} \\
& =\int_{0}^{\infty} d t_{1} \ldots d t_{n}\left[\lim _{\alpha \rightarrow \infty} \alpha^{h_{1}+\ldots+h_{n}-n} f\left(\frac{t_{1}}{\alpha}, \ldots, \frac{t_{n}}{\alpha}\right)\right] e^{-t_{1} K_{1}} \ldots e^{-t_{n} K_{n}} \\
& =0 .
\end{aligned}
$$

This means that $F\left(K_{1}, \ldots, K_{n}\right)$ vanishes at $K_{i} \rightarrow \infty$ faster the inverse power of $h_{1}+\ldots+h_{n}$. Therefore, if $\Phi$ admits a dual $\mathcal{L}^{-}$level expansion, the leading level must be strictly negative.

One corollary of this result is that solutions cannot have zero or positive levels in the dual $\mathcal{L}^{-}$level expansion, since the action and closed string overlap [26] must be welldefined. Usually it is easy to check this by reading off the leading behavior of the solution 
around $K=\infty$. For example, let's look at the "simple" tachyon vacuum [9] and the KOS marginal solution [13]:

$$
\begin{aligned}
\Psi_{\text {simple }} & =(c+Q(B c)) \frac{1}{1+K}, \\
\Psi_{\mathrm{KOS}} & =\frac{1}{\sqrt{1+K}} Q \sigma_{01} \frac{B}{1+K} \sigma_{10}(1+K) c \frac{1}{\sqrt{1+K}} .
\end{aligned}
$$

Expanding around $K=\infty$ gives the leading behavior:

$$
\begin{aligned}
\Psi_{\text {simple }} & =Q(B c) \frac{1}{K}+\ldots \\
\Psi_{\mathrm{KOS}} & =\frac{1}{\sqrt{K}}\left[Q \sigma_{01} \frac{B}{K} Q \sigma_{10}+\sigma_{01} Q \sigma_{10}\right] \frac{1}{\sqrt{K}}+\ldots .
\end{aligned}
$$

Recalling that $c$ has scaling dimension -1 and the boundary condition changing operators $\sigma_{01}$ and $\sigma_{10}$ have dimension 0 , we can see that the leading level for both solutions is -1 . This is the highest possible (integer) level consistent with a regularity, so in a sense these solutions are as identity-like as possible. There are also well-known identity-based solutions for the tachyon vacuum $[10,11]$ and marginal deformations $[27]$

$$
\Psi_{\mathrm{tv}}=c(1-K), \quad \Psi_{\operatorname{marg}}=c V .
$$

Since the marginal operator $V$ has dimension 1, the leading level for both of these solutions is level 0 , which means that they are too identity-like.

Let us mention one other result, which is important for the physical interpretation of the dual $\mathcal{L}^{-}$level expansion:

Theorem 3.3. Let $\Psi$ be a regular solution which admits a dual $\mathcal{L}^{-}$level expansion. Then all finite levels in the dual $\mathcal{L}^{-}$level expansion of $\Psi$ can be eliminated by a gauge transformation.

Proof. The dual $\mathcal{L}^{-}$level expansion of $\Psi$ takes the form

$$
\Psi=\Psi_{h}+\text { lower levels, }
$$

where $\frac{1}{2} \mathcal{L}^{-} \Psi_{h}=h \Psi_{h}$ and "lower levels" denotes a sequence of $\mathcal{L}^{-}$eigenstates with eigenvalue less than $h$. Since by assumption $\Psi$ is a well-defined solution, theorem 3.2 implies that $h$ is less than zero. Now consider the level $h$ contribution to the equations of motion $Q \Psi+\Psi^{2}=0$. Since $h$ is negative, the highest level of $\Psi^{2}$ is necessarily less than $h$, so the quadratic term does not contribute at this level. Therefore the equations of motion imply

$$
Q \Psi_{h}=0
$$


Now we want to explicitly remove the term $\Psi_{h}$ by a gauge transformation. Assume the gauge transformation takes the form

$$
U=1+U_{h}+\text { lower levels. }
$$

Thus we require that $U^{-1}(Q+\Psi) U$ vanishes at level $h$, which implies

$$
Q U_{h}+\Psi_{h}=0
$$

We can solve this equation only if $\Psi_{h}$ is BRST exact. However, at finite levels all BRST closed states are exact, since we can define the homotopy operator

$$
A=\frac{B}{K}
$$

Of course this is a singular state, but according to the current philosophy this is not a problem. The sliver divergence can be arranged to cancel against lower level states. Therefore we can remove the leading level term $\Psi_{h}$ with a gauge transformation of the form

$$
U=1-\frac{B}{K} \Psi_{h}+\text { lower levels. }
$$

We assume that the lower level states can be chosen so that $U$ is well-defined and invertible. This means that the highest level of $\Psi$ can be eliminated by a gauge transformation, and by repeating this process iteratively, all finite levels can be eliminated.

This result means that there is no gauge invariant information revealed by the dual $\mathcal{L}^{-}$ level expansion. In a sense, there is no "physics" in the identity string field. All of the physics of is carried by states at level $-\infty$. Note that the prototypical example of a level $-\infty$ state is the sliver; Therefore, theorem 3.3 fits well with studies of the phantom term $[14,8]$ and vacuum string field theory [28, 29, 30], which suggest that all of the physics of open string field theory can be encoded in finite rank projectors.

Let illustrate the method behind the proof of theorem 3.3 with an example. Suppose that for some application the "simple" tachyon vacuum solution is too identity-like, and we want to remove the leading term in the dual $\mathcal{L}^{-}$level expansion. The leading term is written in (3.29). Plugging into (3.37) we find that the required gauge transformation takes the form

$$
U=1-\frac{B}{K} Q(B c) \frac{1}{K}+\ldots=1-B c \frac{1}{K}+\ldots .
$$

To complete the definition of $U$ we should fix the lower level states according to our convenience. The first priority is to get rid of the pole at $K=0$. This can be achieved, for example, by replacing $\frac{1}{K}$ with $\frac{1}{K+1}$, but unfortunately the resulting gauge transformation is not invertible. So instead we can replace $\frac{1}{K}$ with $\frac{1}{K+2}$ :

$$
U=1-B c \frac{1}{K+2} \text {. }
$$


Transforming the "simple" tachyon vacuum with this gauge parameter happens to give the solution

$$
\begin{aligned}
\Psi^{\prime} & =U^{-1}\left(Q+\Psi_{\text {simple }}\right) U \\
& =\frac{K+2}{K+1}\left[c \frac{B}{K+2} c+Q(B c)\right] \frac{1}{(K+1)(K+2)} .
\end{aligned}
$$

The dual $\mathcal{L}^{-}$level expansion takes the form

$$
\Psi^{\prime}=Q(B c) \frac{1}{K^{2}}+\ldots
$$

Now the leading level is -2 . The level -1 state of the "simple" tachyon vacuum has been removed as desired.

\section{Applications to Solutions in the $K B c$ subalgebra}

In this section we turn our attention to solutions in the $K B c$ subalgebra. The string fields $K$ and $B$ were discussed before. The field $c$ corresponds to a local insertion of the $c$ ghost in correlation functions on the cylinder. It satisfies the identities

$$
c^{2}=0, \quad c B+B c=1, \quad Q c=c K c,
$$

and has scaling dimension -1 :

$$
\frac{1}{2} \mathcal{L}^{-} c=-c .
$$

Equations (4.1) and (2.3) define what we call the $K B c$ subalgebra. Note that $c$ satisfies additional relations which are not implied by the $K B c$ subalgebra, for example $(\partial c)^{2}=0$ (using the notation $\partial \equiv[K, \cdot]$ ). We call these auxiliary identities. ${ }^{6}$ A general realization of the $K B c$ subalgebra does not satisfy these identities. See, for example, equation (4.11) later. To simplify the discussion we will focus on solutions which satisfy the equations of motion by virtue of the basic relations of the $K B c$ subalgebra, (2.3) and (4.1), alone. In particular, theorems 4.1 and 4.2 apply to solutions in this class. Auxiliary identities produce further solutions which we have not systematically analyzed, but play a role in later discussion.

Our task is to identify all possible gauge orbits of solutions in the $K B c$ subalgebra using the $\mathcal{L}^{-}$level expansion, and then to investigate the regularity of these solutions using the dual $\mathcal{L}^{-}$level expansion. Our results can be summarized as follows: We identify 6 gauge equivalence classes of solutions:

1) Perturbative vacuum

\footnotetext{
${ }^{6}$ The general set of auxiliary identities is $\left(\partial^{m} c\right)^{2}=0$ for all $m \geq 1$. This implies $\partial^{m} c \partial^{n} c=-\partial^{n} c \partial^{m} c$. Equation (4.1) only implies the cases $m=n=0$ and $m=0, n=1$.
} 
2) Tachyon vacuum

3) Residual perturbative vacuum

4) Residual tachyon vacuum

5) Residual conjugate tachyon vacuum

6) MNT ghost brane

The last four types of solution are unexpected. We will call them residual solutions. ${ }^{7}$ As we will see, they are singular from the perspective of the identity string field. In particular, residual solutions always have zero or positive levels in the dual $\mathcal{L}^{-}$level expansion.

Consider the $\mathcal{L}^{-}$level expansion of a solution $\Psi$ and gauge parameter $U$ in the $K B c$ subalgebra:

$$
\begin{gathered}
\Psi=\Psi_{-1}+\Psi_{0}+\Psi_{1}+\Psi_{2}+\ldots, \\
\Psi_{-1} \equiv \alpha c \\
\Psi_{0} \equiv \gamma_{1} c K+\gamma_{2} K c+\beta c K B c, \\
\vdots \\
U=U_{0}+U_{1}+U_{2}+\ldots, \\
U_{0} \equiv 1+\lambda B c \quad(\lambda \neq-1), \\
\vdots
\end{gathered}
$$

The index on the eigenstates $\Psi_{n}$ and $U_{n}$ refers to their $\frac{1}{2} \mathcal{L}^{-}$eigenvalue, and the constants $\alpha, \beta, \gamma_{1}, \gamma_{2}$ are coefficients to be determined by solving the equations of motion. The constant $\lambda$ in $U$ can take any value besides -1 , which is not allowed since $U$ must be invertible. The equations of motion imply

$$
\Psi_{-1}^{2}=0
$$

which is satisfied for any choice of the coefficient $\alpha$ in front of $c$. Transforming $\Psi$ with $U$, it is easy to show that all nonzero choices of $\alpha$ can be related by a gauge transformation with the appropriate choice of $\lambda$. However, $\alpha=0$ and $\alpha \neq 0$ cannot be related by a gauge transformation. Solutions with $\alpha \neq 0$ turn out to describe the tachyon vacuum:

$$
\text { Tachyon vacuum : } \Psi=\alpha c+\ldots \quad(\alpha \neq 0),
$$

\footnotetext{
${ }^{7}$ The residual tachyon vacuum solutions are discussed by Zeze [11]. The residual perturbative vacuum solutions were first pointed out to the author by C. Maccaferri, and they were also noted by MNT [1].
} 
where ... denotes higher level terms. Now let's look at the $\alpha=0$ solutions. The $\mathcal{L}^{-}$level expansion now takes the form

$$
\Psi=\Psi_{0}+\Psi_{1}+\Psi_{2}+\ldots
$$

The equations of motion imply that the leading contribution $\Psi_{0}$ itself satisfies the equations of motion:

$$
Q \Psi_{0}+\Psi_{0}^{2}=0
$$

Plugging in $\Psi_{0}$ from (4.3), straightforward algebra reveals five possible solutions:

$$
\begin{array}{ll}
\text { Perturbative vacuum : } & \Psi=\beta c K B c+\ldots, \quad(\beta \neq-1) \\
\text { Residual perturbative vacuum : } & \Psi=-c K B c+\ldots, \\
\text { Residual tachyon vacuum : } & \Psi=-c K+\ldots, \\
\text { Residual conjugate tachyon vacuum : } & \Psi=-K c+\ldots \\
\text { MNT ghost brane : } & \Psi=-c K-K c+c K B c+\ldots . .
\end{array}
$$

The last four solutions - the residual solutions - are invariant under gauge transformations by $U$ at this level. A gauge transformation of the perturbative vacuum can set the coefficient $\beta$ to any value (with the appropriate choice of $\lambda$ ), except $\beta=-1$. Note that the two residual tachyon vacuum solutions are not gauge equivalent, though they are related by conjugation.

Thus we have extracted a total of six gauge orbits. Now we can ask whether analysis of higher level states will reveal further physically distinct solutions. The answer is no, according to the following theorem:

Theorem 4.1. Equations (4.5) and (4.8) are the only gauge orbits for solutions in the $\mathcal{L}^{-}$level expansion of the $K B$ c subalgebra.

The proof of this statement is somewhat lengthy so we postpone it to appendix A. Note that this theorem only applies to solutions as they are defined in the $\mathcal{L}^{-}$level expansion. Solutions which are not analytic at $K=0$ (for example, multibranes and fractional branes [2]) do not fall within this classification. Moreover, the $\mathcal{L}^{-}$level expansion by itself is not a complete definition of the string field, which should satisfy many regularity conditions which cannot be seen before resummation. Such considerations may exclude certain gauge orbits (for example, as we will argue, the residual solutions) or it can reveal further gauge orbits within the ones already described. The only example of the later phenomenon known to us appears in the so-called half-brane solutions of cubic superstring field theory [25]. We are not aware of such phenomena in the $K B c$ subalgebra.

It is quite surprising to find four distinct solutions in addition to perturbative vacuum and the tachyon vacuum. What do these solutions mean? One basic quantity we can 
calculate is the energy. Actually, there is a rule of thumb which says that the energy (relative to the perturbative vacuum) is the sum of the coefficients of $c K$ and $K c$ in the $\mathcal{L}^{-}$level expansion:

$$
\text { Energy } \sim \gamma_{1}+\gamma_{2}
$$

Thus the residual perturbative vacuum has zero energy, the residual tachyon vacuum has minus the energy of the reference D-brane (i.e. the same energy as the tachyon vacuum), and the MNT ghost brane has minus twice the energy of the reference D-brane. To see where the rule (4.9) comes from, first note that $\gamma_{1}+\gamma_{2}$ is a gauge invariant quantity for all ghost number 1 states in the $K B c$ subalgebra. Therefore, if $\gamma_{1}+\gamma_{2}$ computes the energy for one solution in each gauge orbit, it computes the energy for all solutions. So let us take a representative solution from each gauge orbit which terminates at level 0 in the $\mathcal{L}^{-}$ level expansion:

$$
\Psi=\alpha c+\gamma_{1} c K+\gamma_{2} K c+\beta c K B c
$$

where the coefficients are fixed according to which gauge orbit we are describing. We cannot compute the energy of (4.10) directly since the solution is too identity-like. To fix this problem, we regularize the $K B c$ subalgebra by defining the fields [31]

$$
\begin{aligned}
\hat{K} & \equiv \frac{K}{1+\epsilon K}, \\
\hat{B} & \equiv \frac{B}{1+\epsilon K}, \\
\hat{c} & \equiv c(1+\epsilon K) B c .
\end{aligned}
$$

It is easy to check that $\hat{K}, \hat{B}$ and $\hat{c}$ satisfy the defining relations (4.1) and (2.3) of the $K B c$ subalgebra. ${ }^{8}$ Moreover, the redefined fields $\hat{K}, \hat{B}$ are more regular than $K, B$ in the dual $\mathcal{L}^{-}$level expansion. With this replacement the solution becomes

$$
\begin{aligned}
\hat{\Psi}=\alpha \hat{c}+\gamma_{1} \hat{c} \hat{K}+\gamma_{2} \hat{K} \hat{c}+\beta \hat{c} \hat{K} \hat{B} \hat{c} \\
=-\gamma_{1}\left(\frac{1}{\epsilon} c+Q(B c)\right) \frac{1}{1+\epsilon K}-\gamma_{2} \frac{1}{1+\epsilon K}\left(\frac{1}{\epsilon} c+Q(B c)\right) \\
\quad+\left(\alpha+\frac{\gamma_{1}+\gamma_{2}}{\epsilon}\right) c+\left(\alpha+\gamma_{1}+\gamma_{2}+\beta\right) c K B c .
\end{aligned}
$$

Now we are in a better position to compute the energy. We could compute the action, but it is a little easier to compute the closed string overlap $\operatorname{Tr}_{\mathcal{V}}[\hat{\Psi}]$, which for our purposes

${ }^{8}$ We can generalize (4.11) given any $F(K)$ satisfying $F(0)=1$ by

$$
\hat{K}=1-F, \quad \hat{B}=B \frac{1-F}{K}, \quad \hat{c}=c \frac{K B}{1-F} c
$$


is equivalent. The first two terms in (4.13) are proportional to (reparameterizations of) of the "simple" tachyon vacuum solution [9], and therefore contribute proportionally to the closed string overlap of the tachyon vacuum. The third term does not contribute to the overlap because $c$ has negative dimension. The fourth term is problematic since it is a level 0 state in the dual $\mathcal{L}^{-}$level expansion, and shouldn't have a well-defined trace. However, since $c K B c$ is BRST exact it is natural to assume

$$
\operatorname{Tr}_{\mathcal{V}}[c K B c] \equiv 0
$$

Under this assumption the closed string overlap is

$$
\operatorname{Tr}_{\mathcal{V}}[\hat{\Psi}]=-\left(\gamma_{1}+\gamma_{2}\right) \times \text { tachyon vacuum overlap, }
$$

and therefore the energy is proportional to $\gamma_{1}+\gamma_{2}$, as claimed. This argument is more rigorous for pure gauge and tachyon vacuum solutions, since there it is possible to find representatives of the gauge orbit where $\alpha+\gamma_{1}+\gamma_{2}+\beta$ vanishes, and we don't need to assume anything about the trace of $c K B c$. For residual solutions in the form (4.13) the state $c K B c$ is present. In a moment we will show that all residual solutions have such an identity-like term.

Let us mention a curiosity which raises doubt as to whether residual solutions have well-defined energy. When we computed the solutions in (4.8), we assumed that $K, B$ and $c$ satisfy only the basic identities (2.3) and (4.1). However, if we also account for the auxiliary identity $(\partial c)^{2}=0$, the solution space at level zero is enlarged. The four residual solutions become special cases of a two parameter family of solutions ${ }^{9}$

$$
\Psi_{0}=\gamma_{1} c K+\gamma_{2} K c-\left(1+\gamma_{1}+\gamma_{2}\right) c K B c
$$

for arbitrary $\gamma_{1}$ and $\gamma_{2}$. This means that all four residual solutions are related by marginal deformations, where $\gamma_{1}$ and $\gamma_{2}$ are marginal parameters (see figure 4.1). Therefore residual solutions must have the same energy, in contradiction with our earlier reasoning. It would be interesting to see how this observation can be reconciled with MNT's calculation of the boundary state $[1,33]$.

These problems appear to be related to the fact that residual solutions are too identitylike. However, we have not shown that all residual solutions suffer from this problem. This is the purpose of the following theorem:

Theorem 4.2. Let $\Psi$ be a residual solution in the KBc subalgebra. Then the highest level in the dual $\mathcal{L}^{-}$level expansion of $\Psi$ is zero or positive.

Proof. A solution in the $K B c$ subalgebra takes the general form

$$
\Psi=\int_{0}^{\infty} d t_{1} d t_{2} d t_{3} f\left(t_{1}, t_{2}, t_{3}\right) \Omega^{t_{1}} c \Omega^{t_{2}} B c \Omega^{t_{3}},
$$

\footnotetext{
${ }^{9}$ The first example of a solution utilizing an auxiliary identity was pointed out to the author by M. Schnabl.
} 


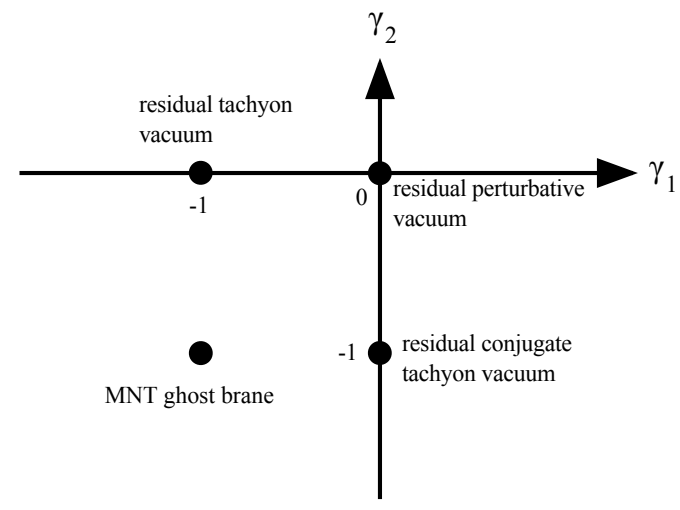

Figure 4.1: Moduli space of residual solutions. The horizontal and vertical axis represent expectation values of the marginal fields $\gamma_{1}$ and $\gamma_{2}$ from (4.16). The four points are the four residual solutions (4.8) whose existence depends only on the basic algebraic relations of the $K B c$ subalgebra. The remaining solutions require the additional relation $(\partial c)^{2}=0$ to satisfy the equations of motion.

where the function $f\left(t_{1}, t_{2}, t_{3}\right)$ is determined by solving the equations of motion. Taking the Laplace transform of $f\left(t_{1}, t_{2}, t_{3}\right)$ gives the function

$$
F\left(K_{1}, K_{2}, K_{3}\right)=\int_{0}^{\infty} d t_{1} d t_{2} d t_{3} f\left(t_{1}, t_{2}, t_{3}\right) e^{-t_{1} K_{1}} e^{-t_{2} K_{2}} e^{-t_{3} K_{3}} .
$$

To make things easier to read we will sometimes omit the $K$ when it appears in the argument of $F$; for example, $F(1,2,3) \equiv F\left(K_{1}, K_{2}, K_{3}\right)$. With a little algebra we can show that the equations of motion imply a functional equation for $F$ :

$$
K_{2} F(1,3,4)-K_{3} F(1,2,4)+F(1,2,2) F(2,3,4)-F(1,2,3) F(3,3,4)=0 .
$$

This equation depends on four variables $K_{1}, K_{2}, K_{3}, K_{4}$. Setting $K_{1}=K_{2}$ and $K_{3}=K_{4}$ we find

$$
\left[K_{2}+F(2,2,2)\right] F(2,3,3)=\left[K_{3}+F(3,3,3)\right] F(2,2,3) .
$$

Let's see what this implies about residual solutions. From (4.8) we find that all four residual solutions have the property

$$
F(K, K, K)=-K+\ldots,
$$

where ... denotes possible higher powers of $K$. This implies, for example,

$$
K_{2}+F(2,2,2)=p K_{2}^{n}+\ldots \quad(n \geq 2),
$$

where $p$ is some constant and $n$ is any power greater than 1 . Note that (4.21) also determines the behavior of $F(2,3,3)$ and $F(2,2,3)$ near $K=0$ :

$$
\begin{aligned}
& F(2,3,3)=a K_{2}-(a+1) K_{3}+\ldots \\
& F(2,2,3)=b K_{2}-(b+1) K_{3}+\ldots
\end{aligned}
$$


where $a, b$ are constants. Plugging these into (4.20), and focusing on the leading term in $K$, gives a constraint of the constants $p, a, b$ from the equations of motion:

$$
p K_{2}^{n}\left(a K_{2}-(1+a) K_{3}\right)=p K_{3}^{n}\left(b K_{2}-(1-b) K_{3}\right) .
$$

When $n \geq 2$, the only solution to this equation is $p=0$. Therefore all higher order corrections in (4.21) vanish, and we have the exact equality:

$$
F(K, K, K)=-K
$$

This means, in particular, that all residual solutions in the $K B c$ subalgebra satisfy the identity

$$
B \Psi B=-B K .
$$

The right hand side is a level 2 state in the dual $\mathcal{L}^{-}$level expansion. Since the highest level of a product of states is less than or equal to the sum of the highest levels of the states individually, this means that the highest level in the dual $\mathcal{L}^{-}$level expansion of any residual solution is zero or positive.

As a final comment, let us mention an oddity related to the characteristic projector [34] of residual solutions. The characteristic projector is the boundary condition changing projector of a singular gauge transformation from a solution to itself [3]. It is expected to give information about the boundary conformal field theory corresponding to a classical solution. The simplest example of a singular gauge transformation from a residual solution to itself takes the form

$$
U=Q_{\Psi_{0}} B=-\left(1+\gamma_{1}+\gamma_{2}\right) B \partial c,
$$

where we take $\Psi_{0}$ from (4.16). Surprisingly, this is exactly the type of singular gauge transformation for which the boundary condition changing projector does not exist. In particular, $U$ is nilpotent, so its kernel and image are not linearly independent and do not define the image and kernel of a projector. One possible interpretation of this result is that the projector does not exist since the solutions do not describe a boundary conformal field theory. In fact, all four residual solutions have physical cohomology in the universal sector (see appendix A), which is difficult to reconcile with a boundary conformal field theory interpretation.

\section{Discussion}

In conclusion, let us discuss the implications of our analysis for the MNT ghost brane solutions. We have shown that these solutions are necessarily singular from the perspective of the identity string field. In the best case scenario, they have an identity-like term of the form

$$
c K B c \text {. }
$$


We claim that such a term renders the action undefined. This requires a little explanation. In particular (5.1) contributes to (for example) the cubic term in the action as

$$
\operatorname{Tr}\left[(c K B c)^{3}\right] .
$$

This appears to vanish without ambiguity both because $c K B c$ is BRST exact and because $(c K B c)^{3}$ is a vanishing state. Nevertheless, a generic regularization of (5.2) does not vanish. Consider for example the regularization

$$
\begin{aligned}
\operatorname{Tr}\left[(c K B c)^{3}\right] & =\lim _{\epsilon \rightarrow 0} \operatorname{Tr}\left[\left(c K B \Omega^{\epsilon t_{1}} c \Omega^{\epsilon t_{2}}\right)^{3}\right] \\
& =\operatorname{Tr}\left[\left(c K B \Omega^{t_{1}} c \Omega^{t_{2}}\right)^{3}\right] .
\end{aligned}
$$

This quantity vanishes if $t_{1}=0$ because $c K B c$ is BRST exact, and it also vanishes if $t_{2}=0$ because $c^{2}=0$, but it does not vanish for generic values of $t_{1}, t_{2}$.

Therefore if we want to define the MNT solution we need to apply some regularization. We can do this, for example, by replacing $c K B c$ with

$$
c K B c \rightarrow c K B c \frac{1}{1+\epsilon K} .
$$

and taking the $\epsilon \rightarrow 0$ limit. This approach appears to be consistent. The equations of motion are satisfied in the Fock space and when contracted with the solution. The situation might be contrasted to solutions with sliver-like singularities [2, 5], where regularization typically produces problems with the equations of motion. What makes the identity-like singularities of MNT more "mild" is that (what might be called) the "dual" sliver state,

$$
(1-\Omega)^{\infty}
$$

vanishes in the Fock space, whereas the sliver state does not. Nevertheless, it seems that the MNT ghost brane and related solutions are not completely healthy. Perhaps further study will clarify whether a regularization such as (5.4) truly defines an acceptable solution.

\section{Acknowledgments}

I would like to thank C. Maccaferri for looking over a draft of the paper, and I. Sachs for kind hospitality in Munich where much of this work was completed. This research was supported by the Grant Agency of the Czech Republic under the grant P201/12/G028.

\section{A Proof of Theorem 4.1}

In this appendix we prove theorem 4.1, which says that any pair of solutions in the $K B c$ subalgebra which share the same leading term in the $\mathcal{L}^{-}$level expansion can be related by 
a gauge transformation. We will prove this by induction; We show that if two solutions are equal up to level $n$, a gauge transformation can make them equal up to level $n+1$. Thus the solutions are gauge equivalent.

Let's start with tachyon vacuum solutions. Suppose we are given two tachyon vacuum solutions which are equal up to level $n$ in the $\mathcal{L}^{-}$level expansion, but differ at level $n+1$ :

$$
\begin{aligned}
\Psi & =\left(\Psi_{-1}+\Psi_{0}+\ldots+\Psi_{n}\right)+\Psi_{n+1}+\ldots, \\
\Psi^{\prime} & =\left(\Psi_{-1}+\Psi_{0}+\ldots+\Psi_{n}\right)+\Psi_{n+1}^{\prime}+\ldots .
\end{aligned}
$$

Our task is to construct a gauge transformation which will make these solutions identical up to level $n+1$. Assume that the gauge parameter takes the form

$$
U=1+U_{n+2}+\ldots
$$

where $U_{n+2}$ is a level $n+2$ state and ... denotes higher level terms. We fix $U_{n+2}$ by requiring that

$$
U^{-1}\left(Q+\Psi^{\prime}\right) U
$$

is equal to $\Psi$ up to level $n+1$. It is automatically equal to $\Psi$ up to level $n$ because of the assumed form of $\Psi^{\prime}$ and $U$. Imposing equality at level $n+1$ gives the equation

$$
\Psi_{n+1}^{\prime}-\Psi_{n+1}=\left[\Psi_{-1}, U_{n+2}\right] .
$$

Note that $\Psi_{-1}$ is nilpotent because it is proportional to $c$. Therefore acting $\left[\Psi_{-1}, \cdot\right]$ on (A.4) implies a condition on $\Psi_{n+1}^{\prime}-\Psi_{n+1}$ :

$$
\left[\Psi_{-1}, \Psi_{n+1}^{\prime}-\Psi_{n+1}\right]=0 .
$$

This condition is implied by the equations of motion, and therefore does not need to be separately assumed. Next we introduce a string field $A_{1}$ at level 1 which satisfies

$$
\left[\Psi_{-1}, A_{1}\right]=1 \text {. }
$$

If $\Psi_{-1}=\alpha c$, then we can take $A_{1}=\frac{1}{\alpha} B$. Taking (A.5) and (A.6) together implies that

$$
U_{n+2}=A_{1}\left(\Psi_{n+1}^{\prime}-\Psi_{n+1}\right)
$$

satisfies (A.4). Therefore all tachyon vacuum solutions, as defined by the leading term in the $\mathcal{L}^{-}$level expansion, are gauge equivalent. Note that this argument does not depend in an essential way on the $K B c$ subalgebra. The higher levels can in principle be composed of more complicated states outside the algebra. All we need is the leading level $\Psi_{-1}$ paired with an operator $A_{1}$ satisfying (A.6).

Now let's consider the five solutions which start at level 0. Assume that a pair of such solutions are equal up to level $n$ :

$$
\begin{gathered}
\Psi=\left(\Psi_{0}+\ldots+\Psi_{n}\right)+\Psi_{n+1}+\ldots, \\
\Psi^{\prime}=\left(\Psi_{0}+\ldots+\Psi_{n}\right)+\Psi_{n+1}^{\prime}+\ldots .
\end{gathered}
$$


We want to find a gauge transformation which makes these solutions identical up to level $n+1$. Assume that the gauge parameter takes the form

$$
U=1+U_{n+1}+\ldots
$$

where $U_{n+1}$ is a level $n+1$ state and ... denotes higher level terms. We fix $U_{n+1}$ by requiring that

$$
U^{-1}\left(Q+\Psi^{\prime}\right) U
$$

is equal to $\Psi$ up to level $n+1$. Equality up to level $n$ follows from the form of $U$ and $\Psi^{\prime}$. Imposing equality at level $n+1$ gives the equation

$$
Q_{\Psi_{0}} U_{n+1}+\Psi_{n+1}^{\prime}-\Psi_{n+1}=0
$$

where $Q_{\Psi_{0}}$ is the kinetic operator around the level 0 solution $\Psi_{0}$. Note that $\Psi_{n+1}^{\prime}-\Psi_{n+1}$ is $Q_{\Psi_{0}}$-closed as a consequence of the equations of motion. We need to show that it is also $Q_{\Psi_{0}}$-exact, so that (A.11) has a solution for $U_{n+1}$.

To prove this, we use a variant of the standard argument that the cohomology of $Q$ is in the kernel of $L_{0}$. To start, consider the BPZ odd component of the $b$-ghost zero mode $\mathcal{B}_{0}$ in the sliver coordinate frame, which we call $\mathcal{B}^{-}$:

$$
\mathcal{B}^{-} \equiv \mathcal{B}_{0}-\mathcal{B}_{0}^{\star}
$$

This is a derivation of the star product and satisfies

$$
\frac{1}{2} \mathcal{B}^{-} K=B, \quad \frac{1}{2} \mathcal{B}^{-} B=0, \quad \frac{1}{2} \mathcal{B}^{-} c=0
$$

Also

$$
\left[Q, \mathcal{B}^{-}\right]=\mathcal{L}^{-}
$$

Now define the operator

$$
\mathcal{L}_{\Psi_{0}}^{-} \equiv\left[Q_{\Psi_{0}}, \mathcal{B}^{-}\right]=\mathcal{L}^{-}+\left[\left(\mathcal{B}^{-} \Psi_{0}\right), \cdot\right]
$$

If $\mathcal{L}_{\Psi_{0}}^{-}$is diagonalizable, then the cohomology of $Q_{\Psi_{0}}$ can be found in its kernel. In particular if $\phi$ is a $Q_{\Psi_{0}}$ closed eigenstate of $\mathcal{L}_{\Psi_{0}}^{-}$with eigenvalue $h \neq 0$, then

$$
\phi=\frac{1}{h} Q_{\Psi_{0}}\left(\mathcal{B}^{-} \phi\right)
$$

so $\phi$ is exact.

So our goal is to identify the states in the kernel of $\mathcal{L}_{\Psi_{0}}^{-}$for positive levels in the $K B c$ subalgebra, and show that they do not lead to cohomology at ghost number 1 . For the 
five solutions at level 0 in $(4.8)$, the operator $\mathcal{L}_{\Psi_{0}}^{-}$takes the form

$$
\begin{array}{ll}
\text { Perturbative vacuum : } & \frac{1}{2} \mathcal{L}_{\Psi_{0}}^{-}=\frac{1}{2} \mathcal{L}^{-}, \\
\text {Residual perturbative vacuum : } & \frac{1}{2} \mathcal{L}_{\Psi_{0}}^{-}=\frac{1}{2} \mathcal{L}^{-}, \\
\text {Residual tachyon vacuum : } & \frac{1}{2} \mathcal{L}_{\Psi_{0}}^{-}=\frac{1}{2} \mathcal{L}^{-}+[c B, \cdot], \\
\text { Residual conjugate tachyon vacuum : } & \frac{1}{2} \mathcal{L}_{\Psi_{0}}^{-}=\frac{1}{2} \mathcal{L}^{-}+[c B, \cdot], \\
\text { MNT ghost brane : } & \frac{1}{2} \mathcal{L}_{\Psi_{0}}^{-}=\frac{1}{2} \mathcal{L}^{-}+2[c B, \cdot] .
\end{array}
$$

For the perturbative vacuum and the residual perturbative vacuum $\mathcal{L}_{\Psi_{0}}^{-}$simply computes twice the level, and therefore has no kernel if the level is positive. For the remaining solutions we need to diagonalize $\mathcal{L}_{\Psi_{0}}^{-}$to see what happens. To do this, note that $\mathcal{L}^{-}$ and $[c B, \cdot]$ commute and so are simultaneously diagonalizable. A standard basis of $\mathcal{L}^{-}$ eigenstates at level $n$ and ghost number 1 in the $K B c$ subalgebra is

$$
K^{p} c K^{q} B c K^{r}, \quad(p+q+r=n+1) .
$$

However, these are not eigenstates of $[c B, \cdot]$. To diagonalize $[c B, \cdot]$ we use an alternative basis of the states at level $n$ :

$$
\begin{array}{llll}
{[c B, \cdot]=1:} & c K^{n+1} B c, & \\
{[c B, \cdot]=0:} & c K^{p} B \partial c K^{q} & & (p+q=n), \\
{[c B, \cdot]=0:} & K^{p} \partial c K^{q} B c & & (p+q=n), \\
{[c B, \cdot]=-1:} & K^{p} \partial c K^{q} B \partial c K^{r} & (p+q+r=n-1) .
\end{array}
$$

In the first three cases, the $[c B, \cdot]$ eigenvalue only adds to the level, so we don't find any states in the kernel. In the last case, however, the $[c B, \cdot]$ eigenvalue subtracts, and there is the possibility of cohomology. For the residual tachyon vacuum solutions this occurs at level 1 and for the MNT ghost brane at level 2. At level 1 there is a single state of the form (A.22):

$$
B(\partial c)^{2}
$$

This state vanishes as a consequence of the auxiliary identity $(\partial c)^{2}=0$, but at any rate is trivial in the cohomology:

$$
(\partial c)^{2} B=Q_{-c K} \partial c B
$$

Now consider the MNT ghost brane. There are three possible elements of the cohomology at level 2:

$$
K(\partial c)^{2} B, \quad B(\partial c)^{2} K, \quad \partial c K B \partial c .
$$


The first two states vanish assuming $(\partial c)^{2}=0$, but are also trivial in the cohomology:

$$
\begin{aligned}
& K(\partial c)^{2} B=Q_{-c K-K c+c K B c} K \partial c B, \\
& B(\partial c)^{2} K=Q_{-c K-K c+c K B c} B \partial c K .
\end{aligned}
$$

The third state $\partial c K B \partial c$ does not vanish, and is not $Q_{\Psi_{0}}$-closed if we assume only the defining relations (2.3) and (4.1) of the $K B c$ subalgebra. However, if we assume auxiliary identities $\partial c K B \partial c$ is a nontrivial element of the cohomology

$$
Q_{-c K-K c+c K B c} \partial c K B \partial c=0, \quad \partial c K B \partial c \neq Q_{-c k-K c+c K B c} \text { (something) }
$$

Therefore $\partial c K B \partial c$ generates a physically nontrivial deformation of the MNT ghost brane background. However, the resulting solutions require auxiliary identities to satisfy the equations of motion, and our more limited goal is to classify solutions which satisfy the equations of motion only by virtue of the defining relations of the $K B c$ subalgebra (2.3) and (4.1). Then for our purposes $\partial c K B \partial c$ is not $Q_{\Psi_{0}}$-closed, and in all cases (A.11) has a solution $U_{n+1}$. This completes the proof.

An byproduct of our proof is a classification of the physical cohomology in the $K B c$ subalgebra, supplemented with auxiliary identities, around all four residual solutions. We list these in table 2. Note that the perturbative vacuum and (of course) the tachyon vacuum have no ghost number 1 cohomology in the $K B c$ subalgebra since there are no on-shell vertex operators in the universal sector.

The reader may ask what can be said about the classification of solutions in the $K B c$ subalgebra additionally assuming the full set of relations satisfied by $c$. In this case we would have to examine the cohomology at positive levels around all of the solutions in (4.16). There is a possibility of enhanced cohomology whenever $\gamma_{1}+\gamma_{2}$ is an integer. Moreover, for these solutions theorem 4.2 would have to be carefully reconsidered, as its proof is based on the functional relation (4.19) which assumes only the basic relations (4.1). Therefore, we have not excluded the possibility that auxiliary identities could produce physically interesting solutions, for example multibranes.

\section{References}

[1] T. Masuda, T. Noumi and D. Takahashi, "Constraints on a class of classical solutions in open string field theory," arXiv:1207.6220 [hep-th].

[2] M. Murata and M. Schnabl, "On Multibrane Solutions in Open String Field Theory," Prog. Theor. Phys. Suppl. 188, 50 (2011) [arXiv:1103.1382 [hep-th]]; "Multibrane Solutions in Open String Field Theory," arXiv:1112.0591 [hep-th].

[3] T. Erler and C. Maccaferri, "Connecting Solutions in Open String Field Theory with Singular Gauge Transformations," JHEP 1204, 107 (2012) [arXiv:1201.5119 [hepth]]. 


\begin{tabular}{|c|c|c|c|c|}
\hline & level -1 & level 0 & level 1 & level 2 \\
\hline $\begin{array}{c}\text { Residual perturbative } \\
\text { vacuum }\end{array}$ & - & $\begin{array}{c}c K B c \\
B c \partial c \\
c \partial c B\end{array}$ & - & - \\
\hline $\begin{array}{l}\text { Residual tachyon } \\
\text { vacuum }\end{array}$ & $c$ & $\begin{array}{l}B c \partial c \\
c \partial c B\end{array}$ & - & - \\
\hline $\begin{array}{l}\text { Residual conjugate } \\
\text { tachyon vacuum }\end{array}$ & $c$ & $\begin{array}{l}B c \partial c \\
c \partial c B\end{array}$ & - & - \\
\hline MNT ghost brane & - & $\begin{array}{l}B c \partial c \\
c \partial c B\end{array}$ & - & $\partial c K B \partial c$ \\
\hline
\end{tabular}

Table 2: Elements of the ghost number 1 cohomology in the $K B c$ algebra (extended with auxiliary identities) around all four residual solutions. We take the kinetic operator $Q_{\Psi_{0}}$ around the level 0 representatives of these gauge orbits. The cohomology element $c$ represents a deformation of the residual tachyon vacuum into the tachyon vacuum. Likewise, $c K B c$ represents a deformation of the residual perturbative vacuum into the perturbative vacuum. The remaining cohomology elements follow from auxiliary identities. The fields $B c \partial c$ and $c \partial c B$ generate marginal deformations (4.16) which connect the residual solutions inside a single moduli space. The field $\partial c K B \partial c$ represents an additional deformation of the MNT ghost brane whose interpretation is unclear.

[4] T. Okuda and T. Takayanagi, "Ghost D-branes," JHEP 0603, 062 (2006). [hepth/0601024].

[5] T. Erler and C. Maccaferri, "Comments on Lumps from RG flows," JHEP 1111, 092 (2011) [arXiv:1105.6057 [hep-th]].

[6] D. Takahashi, "The boundary state for a class of analytic solutions in open string field theory," JHEP 1111, 054 (2011) [arXiv:1110.1443 [hep-th]].

[7] H. Hata and T. Kojita, "Winding Number in String Field Theory," JHEP 1201, 088 (2012) [arXiv:1111.2389 [hep-th]].

[8] T. Erler and C. Maccaferri, "The Phantom Term in Open String Field Theory," arXiv:1201.5122 [hep-th]. 
[9] T. Erler and M. Schnabl, "A Simple Analytic Solution for Tachyon Condensation," JHEP 0910, 066 (2009) [arXiv:0906.0979 [hep-th]].

[10] E. A. Arroyo, "Generating Erler-Schnabl-type Solution for Tachyon Vacuum in Cubic Superstring Field Theory," J. Phys. A 43, 445403 (2010) [arXiv:1004.3030 [hep-th]].

[11] S. Zeze, "Regularization of identity based solution in string field theory," JHEP 1010, 070 (2010) [arXiv:1008.1104 [hep-th]].

[12] E. A. Arroyo, "Comments on regularization of identity based solutions in string field theory," JHEP 1011, 135 (2010) [arXiv:1009.0198 [hep-th]].

[13] M. Kiermaier, Y. Okawa and P. Soler, "Solutions from boundary condition changing operators in open string field theory," JHEP 1103, 122 (2011) [arXiv:1009.6185 [hepth]].

[14] M. Schnabl, "Analytic solution for tachyon condensation in open string field theory," Adv. Theor. Math. Phys. 10, 433 (2006) [arXiv:hep-th/0511286].

[15] Y. Okawa, "Comments on Schnabl's analytic solution for tachyon condensation in Witten's open string field theory," JHEP 0604, 055 (2006) [arXiv:hep-th/0603159].

[16] T. Erler, "Split string formalism and the closed string vacuum," JHEP 0705, 083 (2007) [arXiv:hep-th/0611200].

[17] L. Rastelli and B. Zwiebach, "Tachyon potentials, star products and universality," JHEP 0109, 038 (2001) [hep-th/0006240].

[18] M. Schnabl, "Wedge states in string field theory," JHEP 0301, 004 (2003) [hepth/0201095].

[19] Y. Okawa, L. Rastelli and B. Zwiebach, "Analytic solutions for tachyon condensation with general projectors," [arXiv:hep-th/0611110].

[20] L. Rastelli and B. Zwiebach, JHEP 0801, 020 (2008) [hep-th/0606131].

[21] E. Aldo Arroyo, "The Tachyon Potential in the Sliver Frame," JHEP 0910, 056 (2009) [arXiv:0907.4939 [hep-th]].

[22] E. A. Arroyo, "Cubic interaction term for Schnabl's solution using Pade approximants," J. Phys. A A 42, 375402 (2009) [arXiv:0905.2014 [hep-th]].

[23] D. Takahashi, "The boundary state for a class of analytic solutions in open string field theory," JHEP 1111, 054 (2011) [arXiv:1110.1443 [hep-th]].

[24] T. Erler, "Split string formalism and the closed string vacuum. II," JHEP 0705, 084 (2007) arXiv:hep-th/0612050. 
[25] T. Erler, "Exotic Universal Solutions in Cubic Superstring Field Theory," JHEP 1104, 107 (2011) [arXiv:1009.1865 [hep-th]].

[26] I. Ellwood, "The Closed string tadpole in open string field theory," JHEP 0808, 063 (2008). [arXiv:0804.1131 [hep-th]].

[27] I. Ellwood, "Rolling to the tachyon vacuum in string field theory," JHEP 0712, 028 (2007) [arXiv:0705.0013 [hep-th]].

[28] L. Rastelli, A. Sen and B. Zwiebach, "String field theory around the tachyon vacuum," Adv. Theor. Math. Phys. 5, 353 (2002) [hep-th/0012251].

[29] L. Rastelli, A. Sen and B. Zwiebach, "Classical solutions in string field theory around the tachyon vacuum," Adv. Theor. Math. Phys. 5, 393 (2002) [hep-th/0102112].

[30] L. Rastelli, A. Sen and B. Zwiebach, "Boundary CFT construction of D-branes in vacuum string field theory," JHEP 0111, 045 (2001) [hep-th/0105168].

[31] T. Erler, "A simple analytic solution for tachyon condensation," Theor. Math. Phys. 163, 705 (2010) [Teor. Mat. Fiz. 163, 366 (2010)].

[32] I. Kishimoto and Y. Michishita, "Comments on solutions for nonsingular currents in open string field theories," Prog. Theor. Phys. 118, 347 (2007) [arXiv:0706.0409 [hep-th]].

[33] M. Kiermaier, Y. Okawa and B. Zwiebach, "The boundary state from open string fields," [arXiv:0810.1737 [hep-th]].

[34] I. Ellwood, "Singular gauge transformations in string field theory," JHEP 0905, 037 (2009) [arXiv:0903.0390 [hep-th]]. 